\title{
Artefacto de centelleo en ultrasonido Doppler color: Más que un incomprendido, un signo de utilidad
}

\author{
Drs. Michael Hirsch $S^{(1)}$, Tamara Palavecino $B^{(1)}$, Boris León $R^{(2)}$.
}

1. Residente de Radiología, Centro de Imagenología, Hospital Clínico Universidad de Chile.

2. Radiólogo, Unidad de Radiología Intervencional. Centro de Imagenología, Hospital Clínico Universidad de Chile. Radiólogo Clínica Alemana-UDD.

\section{Twinkling artifact in color Doppler ultrasound: A misunderstood and useful sign}

\begin{abstract}
The scintillation or twinkling artifact is a not fully understood phenomenon that appears in Color Doppler Ultrasound (U.S.) examinations as a rapid alternation of color immediately behind a stationary echogenic object, giving it a false appearance of movement. This sign is useful in some clinical conditions, especially in urolithiasis, since it increases diagnostic accuracy and sensitivity; therefore, every radiologist should be fully acquainted with this radiologic sign.

Keywords: Artifact, Color Doppler ultrasound, Lithiasis, Radiology.
\end{abstract}

Resumen: El artefacto de centelleo en Doppler color es un fenómeno de origen no bien precisado, que se genera detrás de algunas estructuras ecogénicas y que se manifiesta por una alternancia de colores, sin existir movimiento. Este signo es de utilidad en algunas condiciones clínicas, especialmente en la urolitiasis, aumentando la sensibilidad diagnóstica, por lo que debe ser parte del conocimiento de todo radiólogo.

Palabras clave: Artefacto, Litiasis, Radiología, Ultrasonido Doppler color.

Hirsch M. Artefacto de centelleo en ultrasonido Doppler color: Más que un incomprendido, un signo de utilidad. Rev Chil Radiol 2011; 17 (2): 82-84.

Correspondencia : Dr. Michael Hirsch S. / mphirsch@gmail.com

Trabajo recibido el 24 de noviembre de 2010, aceptado para publicación el 17 de marzo de 2011.

\section{Aspecto Imaginológico}

El "artefacto de centelleo" (twinkling artifact), llamado también "artefacto en cola de cometa en color" (color comet-tail artifact) aparece en el ultrasonido (US) con Doppler color como una rápida alternancia del color inmediatamente detrás de un objeto ecogénico estacionario, dando a éste una falsa apariencia de movimiento (Figura 1). También puede ser visto con la señal Power Doppler y con Doppler espectral, donde origina una señal de ensanchamiento espectral heterogéneo compuesto por bandas verticales adyacentes sin forma de onda ${ }^{(1,2)}$. Algunos autores consideran este artefacto como un signo imaginológico, debido a la utilidad para detectar patologías, especialmente litiasis.

\section{Reseña Histórica}

Fue descrito en 1996 por Rahmouni et al., como un signo artefactual generado por un medio altamente reflector, el cual sin estar en movimiento, provocaba señal al Doppler color, desplegando una rápida alternancia entre rojo y azul(3).

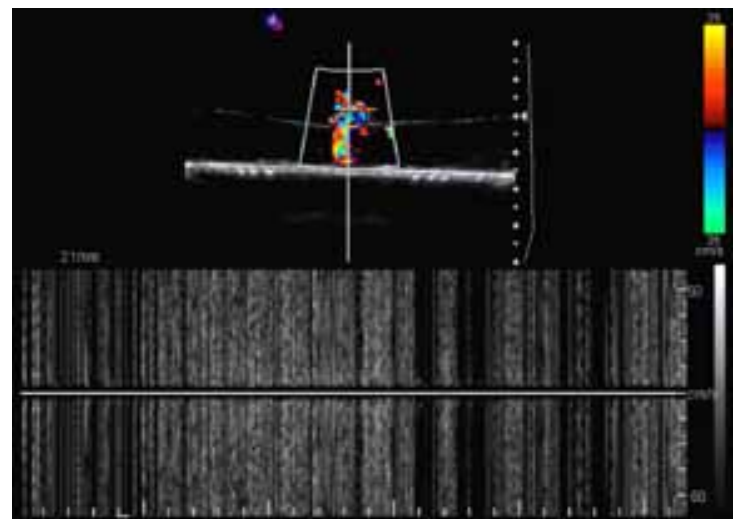

Figura 1. Se ha utilizado una pequeña piedra sumergida en agua, sobre un soporte de gelatina para demostrar el artefacto de centelleo y su representación al Doppler espectral, el cual es característico y consiste en una señal de ensanchamiento espectral heterogéneo compuesto por bandas verticales adyacentes sin forma de onda.

\section{Significado}

La naturaleza del centelleo no está claramente comprendida. Se ha propuesto que la causa subya- 
cente se deba a un ruido de ancho de banda estrecho introducido por fluctuaciones en la fase (o reloj interno) en los circuitos del Doppler del equipo de ultrasonido ${ }^{(1)}$. Se observa especialmente en superficies rugosas, hiperecogénicas, irregulares y con múltiples hendiduras, que provocan una fuerte reflexión de las ondas de ultrasonido incidentes y múltiples reflexiones internas las cuales ensancharían el espectro. A mayor rugosidad de la superficie, mayor el artefacto ${ }^{(1,2)}$. Su apariencia es altamente dependiente del tipo de ecógrafo utilizado y de los parámetros como la frecuencia del transductor, la frecuencia de repetición de pulsos (FRP), la ganancia en escala de grises, la prioridad utilizada y la posición del foco zonal, teniendo relaciones complejas, no lineales, en algunos $\operatorname{casos}^{(1,4)}$. Algunos autores recomiendan aumentar el filtro de pared y la FRP hasta que la representación de los flujos vasculares normales disminuyan, destacando de esta manera sólo el artefacto(2). También se ha demostrado que la posición del foco zonal a nivel de o posterior a la estructura estudiada mejora la visualización del artefacto(4).

Puede ser detectado en áreas calcificadas de diversos tejidos, siendo de gran utilidad en el diagnóstico y seguimiento de diversas entidades clínicas ${ }^{(4)}$.

\section{Diagnóstico Diferencial}

Desde la descripción inicial se ha demostrado su utilidad, principalmente en uro y nefrolitiasis (Figura 2a). Sin embargo, puede ser encontrado en otras patologías como coledocolitiasis y cuerpos extraños, entre otras (Tabla I) ${ }^{(1,2,4)}$.

Tabla I. Entidades que pueden presentar artefacto de centelleo en ecografía.

\begin{tabular}{|ll} 
Vía Biliar & $\begin{array}{l}\text { Litiasis de vía biliar intra y } \\
\text { extrahepática. }\end{array}$ \\
$\begin{array}{l}\text { Riñones y } \\
\text { Vía Urinaria }\end{array}$ & $\begin{array}{l}\text { Litiasis renal y ureteral } \\
\text { Nefrocalcinosis }\end{array}$ \\
Páncreas & $\begin{array}{l}\text { Calcificaciones ductales y } \\
\text { parenquimatosas en } \\
\text { pancreatitis crónica }\end{array}$ \\
Hígado y Bazo & Enfermedades granulomatosas \\
Vesícula Biliar & $\begin{array}{l}\text { Adenomiomatosis } \\
\text { Barro biliar }\end{array}$ \\
& $\begin{array}{l}\text { Calcificaciones pequeñas en la } \\
\text { pared de quistes } \\
\text { Calcificaciones vasculares } \\
\text { Apendicolitos } \\
\text { Clips quirúrgicos } \\
\text { Cuerpos extraños }\end{array}$ \\
\end{tabular}

En el caso de la ureterolitiasis, en un trabajo señalan una sensibilidad de $73.27 \%$ del US en comparación con radiografía, urografía y $\mathrm{TC}^{(5)}$, mientras que otro señalan que el artefacto de centelleo ayuda a confirmar la presencia de pequeños cálculos en un $86 \%$ de los $\operatorname{casos}^{(6)}$. El artefacto de centelleo se presenta en el $95,5 \%$ de los pacientes con nefrolitiasis, versus un $9 \%$ de los controles (pacientes sin historia de urolitiasis, referidos para ecografía abdominal sin patología renal ni urinaria), ayudando en el diagnóstico de cálculos no visualizados en modo $\mathrm{B}^{(7)}$. Esta propiedad es especialmente útil en la litiasis de la unión ureterovesical, en que el uréter puede no estar dilatado, visualizándose incluso jet ureteral al Doppler color, siendo el cálculo pequeño y difícil de visualizar en modo $B$ por la existencia de tejido ecogénico adyacente (Figuras $2 \mathrm{~b}$ y 3$)^{(2)}$.
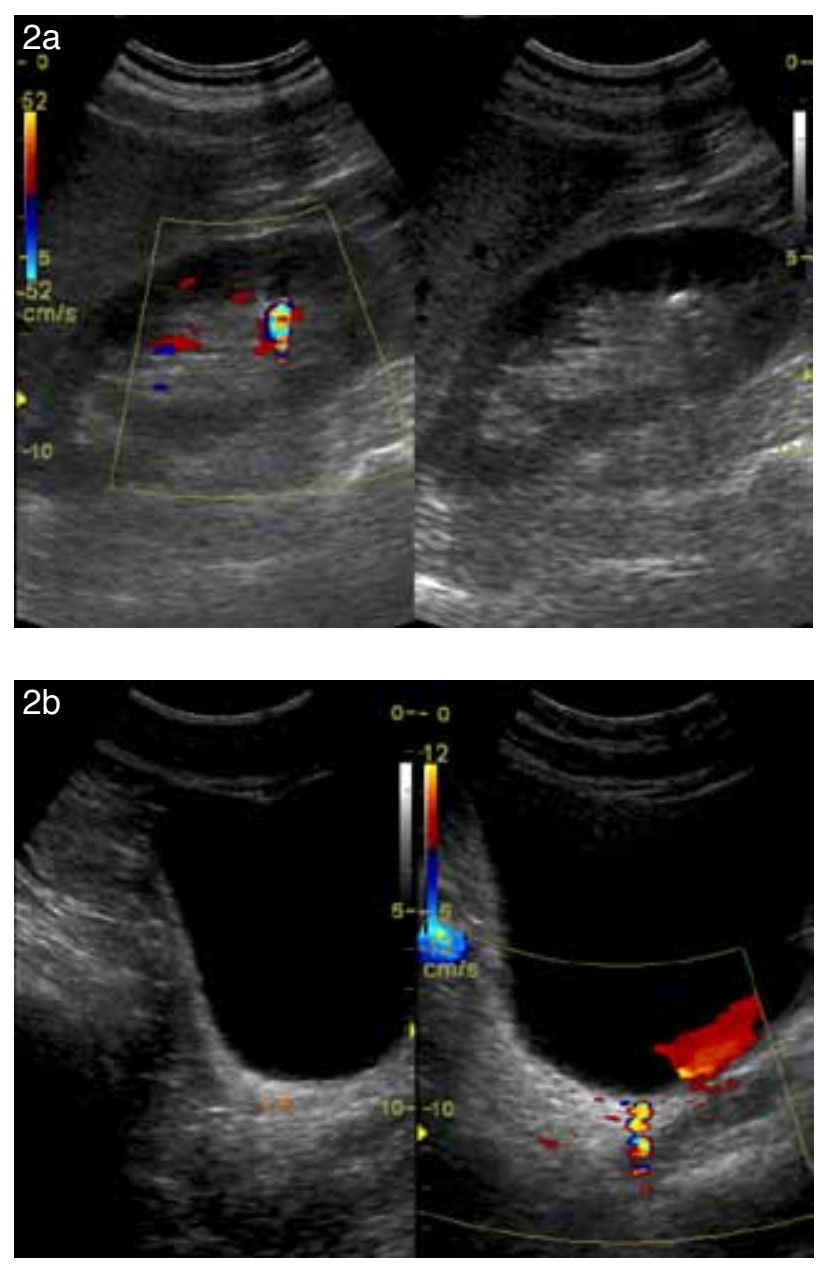

Figura 2. Artefacto de centelleo en nefrolitiasis (a). Se observa imagen ecogénica en seno renal derecho, con escaso artefacto de sombra posterior, cuya naturaleza se hace evidente al Doppler color.

En el mismo paciente se evidencia jet ureteral sin dilatación del uréter (b), hallazgos no sugerentes de urolitiasis. Sin embargo, al Doppler color se evidencia un artefacto de centelleo, con lo que se llega fácilmente al diagnóstico. Cálculo entre calipers. 

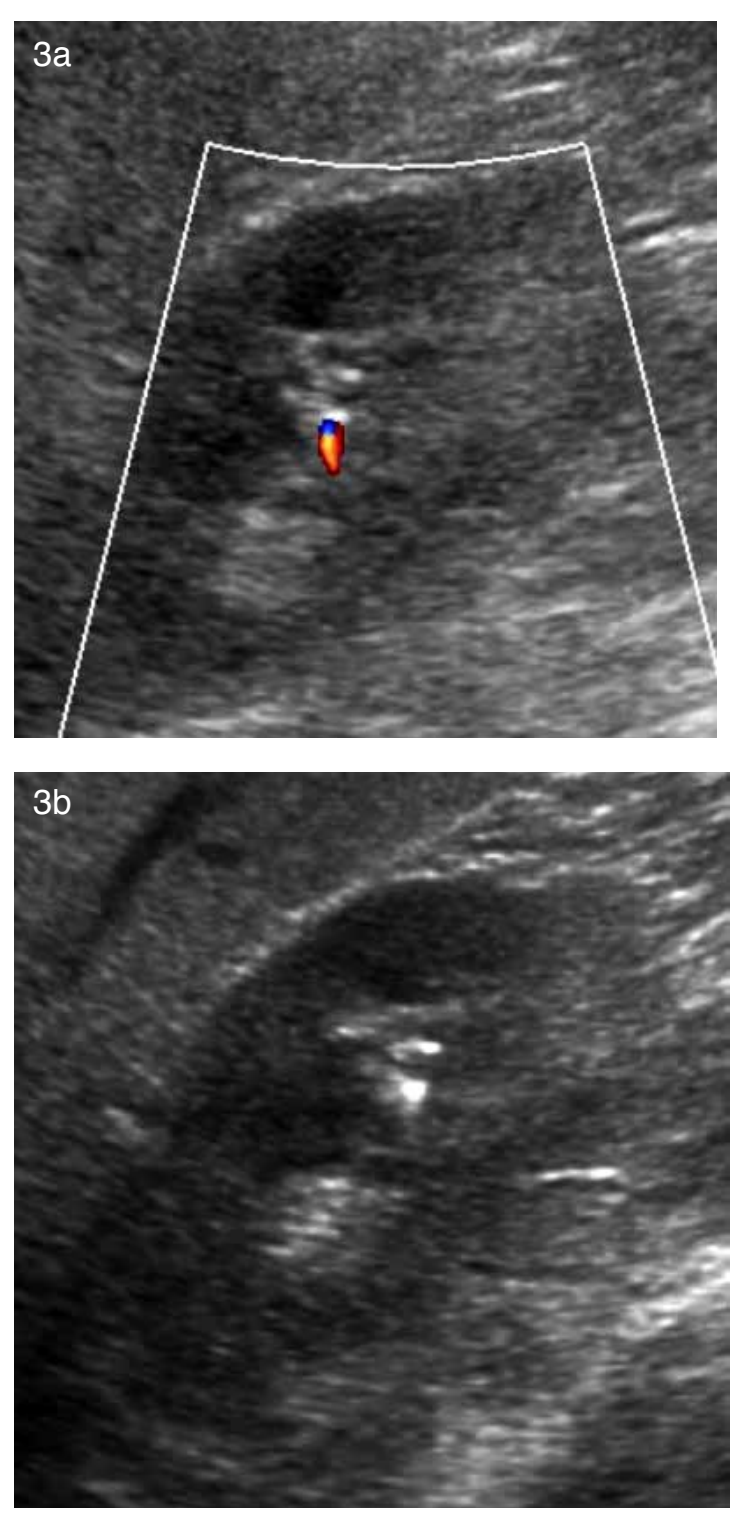

Figura 3. US en que se observa imagen ecogénica en el seno renal derecho, sin artefacto de sombra posterior. Al Doppler color se hace evidente el artefacto de centelleo, el cual pese a aumentar la FRP, se mantiene visible, a diferencia de la vasculatura renal, cuya representación ha desaparecido.

\section{Discusión}

EI US ha comenzado a ocupar cada vez un rol más protagónico en el diagnóstico de patologías como la urolitiasis, debido a su disponibilidad, al ser un método libre de radiación y a la mejoría en la resolución espacial que han tenido los equipos con la introducción de nueva tecnología. Los cálculos son diagnosticados fácilmente cuando tienen distinta ecogenicidad que los tejidos adyacentes y proyectan sombra acústica posterior. Sin embargo, muchos de éstos se encuentran rodeados por tejido ecogénico, como en el seno renal y no generan sombra posterior por su pequeño tamaño, transformándose el artefacto de centelleo en un signo útil en el diagnóstico. Su conocimiento y comprensión permite utilizarlo en las condiciones adecuadas y aumentar la sensibilidad del examen.

\section{Bibliografía}

1. Kamaya A, Tuthill T, Rubin J. Twinkling artifact on color Doppler sonography: dependence on machine parameters and underlying cause. AJR 2003; 180: 215-222.

2. Tchelepi H, Ralls P. Color Comet-Tail Artifact: Clinical Applications. AJR 2009; 192: 11-18.

3. Rahmouni A, Bargoin R, Herment A, Bargoin N, Vasile N. Color Doppler twinkling artifact in hyperechoic regions. Radiology 1996; 199: 269-271.

4. Young Lee J, Hyup Kim S, Yeon Cho J, Han D. Color and Power Doppler Twinkling Artifacts from Urinaary Stones: Clinical Observations and Phantom Studies. AJR 2001; 176: 1441-1445.

5. Mos C, Holt G, luhasz S, Mos D, Teodor I, Halbac M. The sensitivity of transabdominal ultrasound in the diagnosis of ureterolithiasis. Med Ultrason 2010; 12(3): 188-197.

6. Park SJ, Yi BH, Lee HK, Kim YH, Kim GJ, Kim HC Evaluation of patients with suspected ureteral calculi using sonography as an initial diagnostic tool: how can we improve diagnostic accuracy? J Ultrasound Med 2008; 27(10): 1441-1450.

7. Turrin A, Minola P, Costa F, Cerati L, Andrulli S, Trinchieri A. Diagnostic value of colour Doppler twinkling artefact in sites negative for stones on $B$ mode renal sonography. Urol Res 2007; 35(6): 313-317. 